\title{
Investigation of fMRI protocol for evaluation of Gestural Interaction applied to upper-limb motor improvement
}

\author{
Alexandre Fonseca Brandão \\ Institute of Physics Gleb Wataghin \\ Brazilian Institute of Neuroscience and \\ Neurotechnology \\ Campinas, Brazil \\ abrandão@ifi.unicamp.br \\ Gilda Aparecida de Assis \\ Federal University of Ouro Preto \\ João Monlevade, Brazil \\ gildaaa1@gmail.com \\ Gabriela Castellano \\ Institute of Physics Gleb Wataghin \\ Brazilian Institute of Neuroscience and \\ Neurotechnology \\ Campinas, Brazil \\ gabriela@ifi.unicamp.br
}

\author{
Raphael Fernandes Casseb \\ School of Medical Sciences \\ Brazilian Institute of Neuroscience and \\ Neurotechnology \\ Campinas, Brazil \\ rfcasseb@gmail.com \\ Alline Camargo \\ School of Medical Sciences \\ Brazilian Institute of Neuroscience and \\ Neurotechnology \\ Campinas, Brazil \\ camargoalline@live.com
}

\author{
Sara Regina Meira Almeida \\ School of Medical Sciences \\ Brazilian Institute of Neuroscience and \\ Neurotechnology \\ Campinas, Brazil \\ sa.almeida2@gmail.com \\ Li Li Min \\ School of Medical Sciences \\ Brazilian Institute of Neuroscience and \\ Neurotechnology \\ Campinas, Brazil \\ $\underline{\text { limin@fcm.unicamp.br }}$
}

\begin{abstract}
The use of Virtual Reality (VR) systems for rehabilitation treatment as a complement to conventional therapy has grown in recent years. Upper limbs therapy using VR has already been shown useful for stroke patients. In this work, we present a pilot study aiming to investigate the use of a functional magnetic resonance imaging (fMRI) protocol to analyze brain connectivity changes in subjects undergoing upper limb training through a VR environment. Thirteen healthy subjects underwent resting-state fMRI exams before and after a VR session. Although no significant changes are expected in healthy subjects performing only one training session, this study could pave the way for future studies performed with both stroke patients or athletes performing more sessions. Indeed, no significant changes in motor cortex connectivity were found. Nonetheless, an evaluation protocol for this type of VR rehabilitation procedure was successfully established, to be used in further studies with patients or athletes.
\end{abstract}

Keywords - Virtual Reality, Motor stimulus, Healthy subjects, functional Magnetic Resonance Imaging

\section{INTRODUCTION}

Motor and cognitive activities are constantly stimulated in our everyday experiences. Virtual Reality (VR) aims to simulate such situations in virtual environments, and thus it can: provide technical training with reduced risk; improve the teaching-learning process; and provide neuro-motor rehabilitation therapies and new forms of entertainment [1], [2].

The application of VR engines to deliver motor and cognitive stimuli for a particular therapy can be characterized either as exergames, when associated with increased physical activity, or as serious games, when related to technical training or rehabilitation processes. Serious games attempt to simulate practical situations, aiming to provide training for professionals at risk, education in healthcare and business, or even aid the rehabilitation process of several types of patients [3].
The use of VR in therapies has grown, and new forms of interaction with the computer contribute to this process. Sveistrup et al. [4] compared conventional physiotherapy and the use of VR in rehabilitation exercises (shoulder joint) in patients with chronic frozen shoulder, and in rehabilitation exercises for balance in post head trauma patients. They concluded that VR is a safe and motivating therapy. Grealy et al. [5] also investigated the impact of VR in cognitive rehabilitation of head trauma patients and found a significant improvement in reaction times after a four-week intervention.

The effect of treatment using VR applications for recovery of cognitive function, gait, balance and daily life activities was evaluated in stroke patients in [6]. The results of this study showed a lack of evidence in demonstrating meaningful answers regarding the use of VR applications when compared to the responses obtained in conventional therapy. On the other hand, the effectiveness of VR associated with conventional therapy in the treatment of upper limb in patients who suffered a stroke in different levels of motor severity was demonstrated in [7].

Kubicki et al. [8] evaluated if the use of VR could improve the performance of daily life activities, particularly the coordination of general movements and posture of the elderly. The study showed a significant improvement in hands' control, tasks' speed and anticipatory postural adjustment. These results suggest that in the elderly with low functional reserve, some level of motor relearning is maintained. Another study [9] compared the use of VR and real activity for patients with Parkinson's disease, for interaction with fixed and mobile targets, demonstrating that visual stimuli, such as a virtual environment, can improve movement speed and increase the response when the target is moving fast. In [10], VR was employed to simulate daily situations of individuals with cognitive and physical limitations, through multisensory stimuli, in order for them to relearn movements. 
In yet another work [11], aiming at rehabilitation of the upper limbs associated with a VR system, the authors developed two tracking systems, which allowed recording data from the shoulder, elbow, wrist and fingers' motions, to analyze the rehabilitation progress.

In short, these results show that VR systems' inclusion in rehabilitation therapies has potential to improve those, and given VR's intrinsic computerized nature, these systems can also provide data about the rehabilitation progress that would be otherwise difficult, if not impossible, to obtain.

In this paper, we propose to analyze the brain of subjects undergoing VR rehabilitation, using resting-state functional magnetic resonance imaging (rs-fMRI), before and after interaction with a VR environment devised for upper limb motor improvement. The VR rehabilitation system was previously developed by our group [12]. The idea was to test the feasibility of using the rs-fMRI technique as an additional way of evaluating the effect of the rehabilitation procedure. Also, in this way, we could provide a better understanding of how VR rehabilitation systems, specifically, for upper limb rehabilitation, affect the brain of users undergoing therapy. We applied this methodology to healthy subjects, who were evaluated before and after performing one VR training session. Although no significant changes are expected in healthy subjects performing just one training session, this study could pave the way for future studies undertaken with either stroke patients or even athletes performing more sessions. A secondary objective, towards evaluating the use of the VR rehabilitation system [13], was to measure the range of motion (ROM) of the upper limbs.

\section{MATERIALS AND METHODS}

To provide interaction with VR environments we used: (I) the Kinect [14] device (PrimeSense) as a gesture recognition hardware; (II) the OpenNI [15] framework, which allows interaction between hardware and VR software; and (III) the middleware Nite [16], which transforms the input signals (gestural interaction) into output signals, allowing interface control through upper limb movements.

The Kinect device is capable of capturing depth data (3D) in real time from an InfraRed (IR) sensor, and it recognizes if there is a human body in the environment by analyzing silhouettes of objects. The recognition occurs in two stages: first, the shape of a person is identified by the sensor that distinguishes it from other objects present in the same environment; and second, the device system assigns spatial coordinates to body segments of the identified person, to enable the control of a virtual environment [17].

\section{A. Open Natural Interaction - OpenNI}

OpenNI [15] (Open Natural Interaction) is a nonprofit organization formed by the industry manufacturers to certify and promote the compatibility and interoperability of natural interaction devices. By default, it does not allow the use of Kinect, but this is made possible through the Avin2Sensor driver [18]. OpenNI is a framework that provides an application programming interface (API) to develop applications that make use of natural interaction. This API covers communication with low-level devices (vision sensors and audio), as well as high-level solutions (visual tracking using computer vision). The framework is written and distributed under the GNU Lesser General Public License (LGPL), and the source code is freely distributed and available to the general public.

\section{B. Natural Interaction Middleware - NiTE}

NITE [16] (Natural Interaction Middleware) is a middleware used by the OpenNI framework. This middleware is responsible for handling user input obtained by the OpenNI framework and transcribing it into gestures. The middleware provides two types of screening, one for the hands (able to detect gestures such as push, wave, and circle) and another for the body (able to track the major joints of the body).

\section{Virtual Reality Environment: GestureChair and GesturePuzzle}

VR applications (GestureChair and GesturePuzzle, [12], [19]) were chosen to offer motor stimuli for the upper limbs and trigger the activity of efferent (motor) pathways. The aim was to stimulate the execution of the motor task required for the control of each application, detailed below.

The GestureChair application was developed in Java, it is responsive to sensor gestures (recognized by the Kinect device) and based on the KapMan game - Ubuntu software package (this is a free version of the 8-bit Pacman game). In this application, the user controls the game character with hand movements, recognized by the gesture recognition sensor (instead of using the keyboard) (Figure 1).

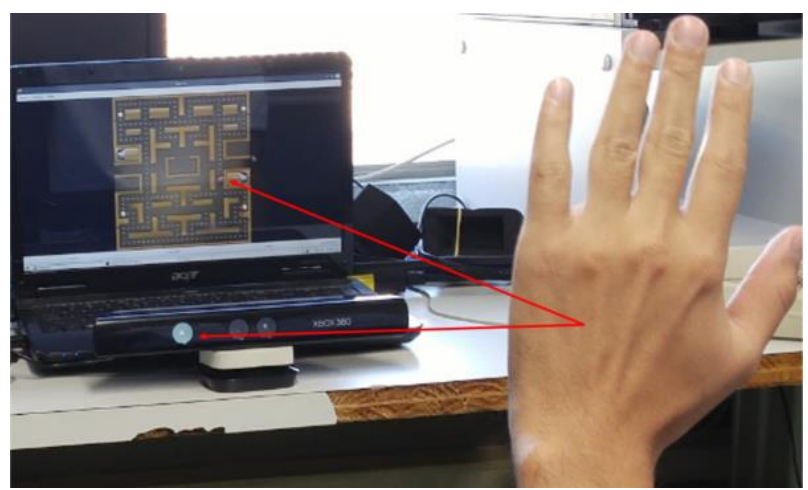

Fig. 1. GestureChair interface is running from gestural interaction.

The recognition of the user's hand wave movement (Figure 1) indicates the start. From this point onwards, the program recognizes every hand gesture and allows the user to control the game. If the initial wave movement is slow, the program does not start interpreting gestures, preventing recognition of unwanted signs.

After started, quick hand movements are required to control the game: (1) elbow flexion (to move the KapMan up, Figure 2a), (2) elbow extension (to move the KapMan down, Figure 2b), (3) external shoulder rotation with the elbow flexed to $90^{\circ}$ (to move the KapMan to the right, Figure 2c) and (4) internal shoulder rotation, also with the elbow flexed to $90^{\circ}$ (to move the KapMan to the left, Figure 2d). 


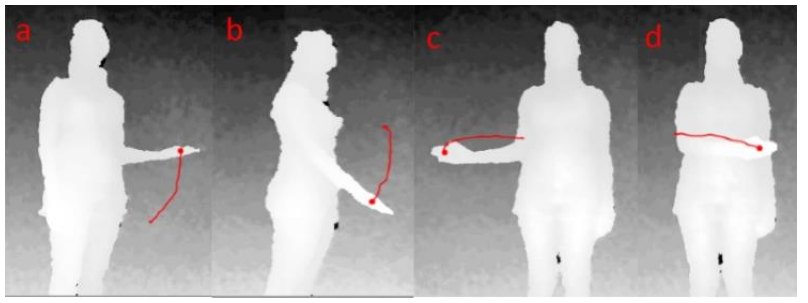

Fig. 2. Movements used to control GestureChair: (a) elbow flexion, (b) elbow extension, (c) lateral rotation of the shoulder with the elbow flexed and (d) medial rotation of the shoulder with the elbow flexed.

The GesturePuzzle application, also developed in Java and responsive to a gesture recognition sensor (Kinect device), consists of a puzzle game in which the movement of the pieces occurs according to upper-limb gestures. The 3D (three-dimensional) coordinates of the player's hand are processed by the application and sent to the game, in order to move the pieces to the correct positions.

The array where the parts are to be placed is located on the left side of the screen, and the shuffled pieces are on the right side (Figure 3). To mount the puzzle, the player must place the hand over one of the parts and move it to the grid. The user also has the option of starting a new game/puzzle with a different image, by positioning his/her hand over the "next image" label.

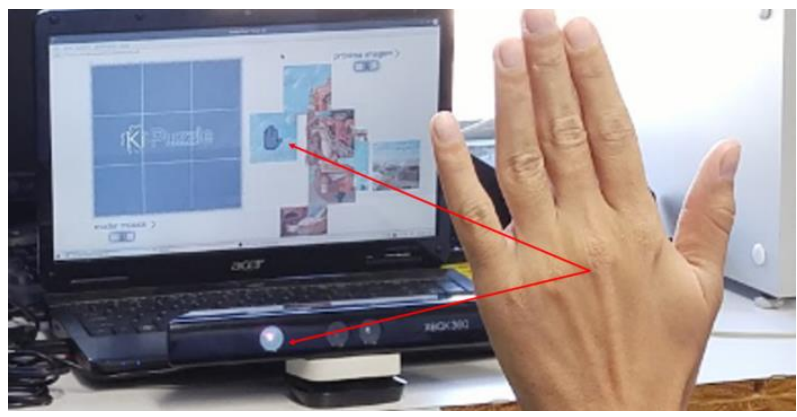

Fig. 3. GesturePuzzle interface is running from gestural interaction.

Upper limb movements can be explored at many levels and ranges of motion (coronal, sagittal and transverse Figure 4), allowing control from both the standing or sitting position.

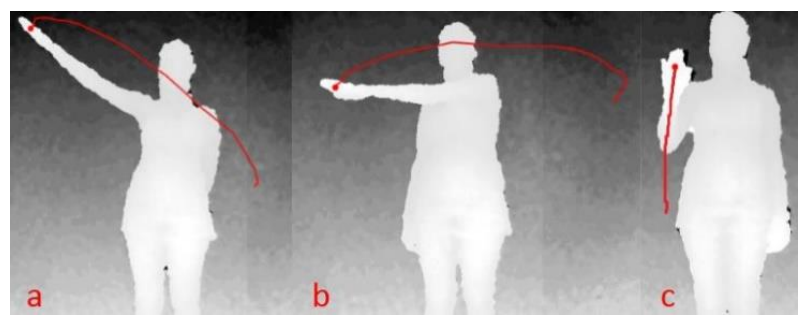

Fig. 4. Movements used to control the GesturePuzzle application: (a) diagonal, (b) transverse and (c) sagittal.

If the user presents a limitation of the upper limbs motion, it is possible to reduce the range of movement by approximating the sensor to the user and, consequently, a shorter action is required to move the pieces on the interface.
The GestureChair and GesturePuzzle applications [12] were chosen due to the recruitment of the elbow and glenohumeral (shoulder) joint for gestural interaction. The movement of these joints is required to prevent muscle atrophy in this region over time. Therefore, the search for new strengthening and rehabilitating techniques constitutes a tremendous social interest.

\section{Subjects and experimental protocol}

Thirteen healthy subjects (6 men, mean age $44 \pm 13$ years) participated in the study. The study was approved by the Ethics Committee on Human Research of University of Campinas (CAAE 35771314.4.0000.5404). All subjects signed an informed consent form before data collection.

Subjects performed one training session with GestureChair and GesturePuzzle, of 30 minutes duration (15 min with GestureChair and 15 min with GesturePuzzle). A physiotherapist, who explained to them how the applications worked, accompanied them. All subjects used their right hand to interact with the VR environment.

Rs-fMRI [20] scans were performed to evaluate brain activity before and after gestural interaction with the VR environment. A seed-based approach was employed to produce correlation maps between a region of interest (ROI) in the primary motor cortex and all other voxels in the brain and tested for significant differences between images of preand post-VR interaction.

\section{E. Image processing and analysis}

Subjects were scanned in a Philips Achieva 3T MRI scanner following a resting state protocol (duration of $6 \mathrm{~min}$, eyes closed).

Anatomical images [T1-weighted, voxel size $=1 \times 1 \times 1$ $\mathrm{mm}^{3}$; image matrix $=240 \times 240 \times 180$; repetition time $(\mathrm{TR})=$ $7.7 \mathrm{~ms}$, echo time $(\mathrm{TE})=3.1 \mathrm{~ms}$; flip angle $\left.=8^{\circ}\right]$ were acquired for every subject, as well as pre and post-VR RS images (T2*-weighted EPI, voxel size $=3 \times 3 \times 3 \mathrm{~mm}^{3}$, image matrix $=80 \times 80 \times 40$, gap $=0.6 \mathrm{~mm}, \mathrm{TR}=2000 \mathrm{~ms}, \mathrm{TE}=30$ $\mathrm{ms}$, ascending acquisition). Preprocessing steps were carried out in SPM 12, which runs within Matlab (Matlab 7.10, MathWorks $\left.{ }^{\circledR}\right)$, in a Windows system. The preprocessing steps for fMRI were: (I) motion correction (rigid body transformation); (II) registration between anatomical and functional images; (III) segmentation into gray matter (GM), white matter (WM) and cerebrospinal fluid (CSF); (IV) normalization to the Montreal Neurological Institute (MNI) template [21]; (V) smoothing with a Gaussian kernel $(\mathrm{FWHM}=6 \mathrm{~mm})$; (VI) removal of linear trends; (VII) bandpass filtering $(0.008-0.1 \mathrm{~Hz})$; (VIII) regression of nuisance signals $(6$ motion parameters + CSF signal + WM signal). Structural images were homogeneity-corrected, segmented into GM, WM, and CSF, and normalized to the MNI space.

To analyze the rs-fMRI data, the WFU PickAtlas toolbox [22] was used to select MNI coordinates for a point within the primary motor cortex, known as Brodmann area 4 (MNI: $58,-14,32)$ and to create a cubic ROI of $5 \times 5 \times 5 \mathrm{~mm}^{3}$ centered at the chosen point (Figure 5a). The spatial average of the time courses within the ROI was extracted for each subject, and Pearson's correlation between this ROI and all the voxels in the brain was computed. Only positive correlation values were considered for the analysis. 
Correlation maps were converted to z-score maps (Fischer ztransformation), and the results were masked to select the voxels within each precentral gyrus (Figure 5b). Finally, a paired t-test was conducted to compare the average $\mathrm{z}$-values for left and right precentral gyri (Figure $5 b$ ), between pre and post conditions.
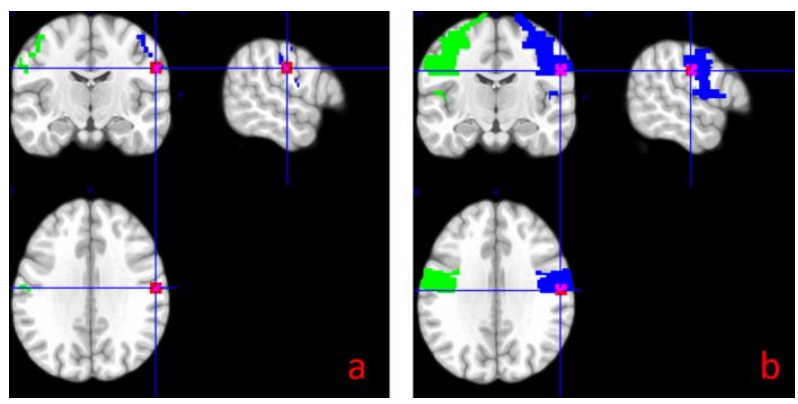

Fig. 5. (a) Cubic ROI centered in the MNI coordinates 58, -14, 32 (red/pink), and the Brodmann area 4 (left - green; right - blue). (b) Same ROI (red/pink) and precentral masks (left - green; right - blue).

\section{RESULTS AND DISCUSSION}

Figure 6 shows the correlation maps obtained between the whole brain and the chosen ROI before (Figure 6a) and after (Figure 6b) the VR interaction. The t-test results obtained for left and right precentral gyri comparing pre and post VR interaction were 1.2260 and 0.8219 , respectively. Although the results between pre and post conditions are visually quite different (as seen in Figure 6), these differences were statistically non-significant $(\mathrm{p}>0.05)$.
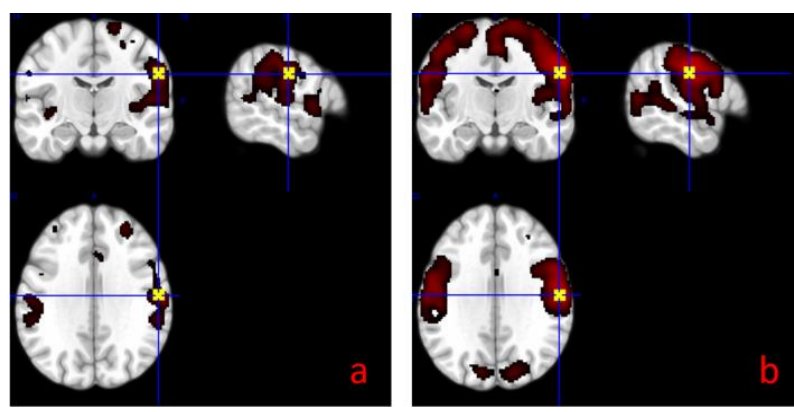

Fig. 6. Correlation maps of the brain with the selected seed (marked with the yellow $\mathrm{x}$ ) for pre (a) and post (b) VR interaction, thresholded at $\mathrm{r}=0.3$ $(\mathrm{z}=0.31)$

The fact that the results found were not significant is not unexpected, since subjects performed only one VR session, which is probably not enough to produce detectable connectivity changes in the brain motor network. Moreover, these were healthy subjects, without any apparent motor disability. On the other hand, the used VR applications have been developed having in mind motor rehabilitation of upper limbs of stroke patients. Patients undergoing rehabilitation therapy usually perform many sessions a week during months. In such cases, we would probably be able to detect some change. Therefore, the present results can serve as a control database to help for comparison with future tests in stroke patients. Also, the rs-fMRI protocol used seems feasible as a tool for evaluation of brain changes resulting from the VR rehabilitation therapy.

In addition to the brain connectivity analysis, the range of motion (ROM) of the upper limbs was measured with the RehabGesture software [13]. Figure 7 presents a histogram with the ROM values of the right elbow joint during interaction with the GestureChair VR software, where it is possible to identify ROM between 60 and 88 degrees (marked in red) as the most requested ROM interval in this joint.

\section{Right Elbow Angle}

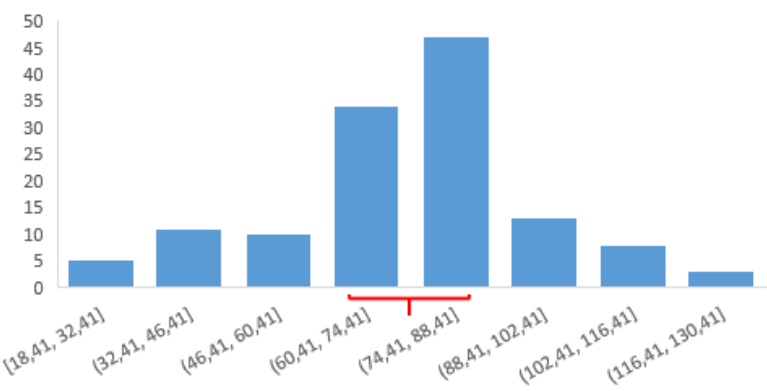

Fig. 7. Histogram of ROM interval for the right elbow flexion, from a single individual, during interaction with the GestureChair application (yaxis: represents how many times a certain range of motion was executed and $\mathrm{x}$-axis: represents the interval in degrees of the range of motion).

In Figure 8, the ROM of the left shoulder joint is presented during interaction with the GesturePuzzle VR software, where it is possible to identify a movement of up to approximately 60 degrees of abduction of the shoulder, needed to complete each of the nine pieces of the virtual puzzle. The highlights in red indicate good motor control and faster execution, while the highlights in blue indicate the need for more time to complete the task.

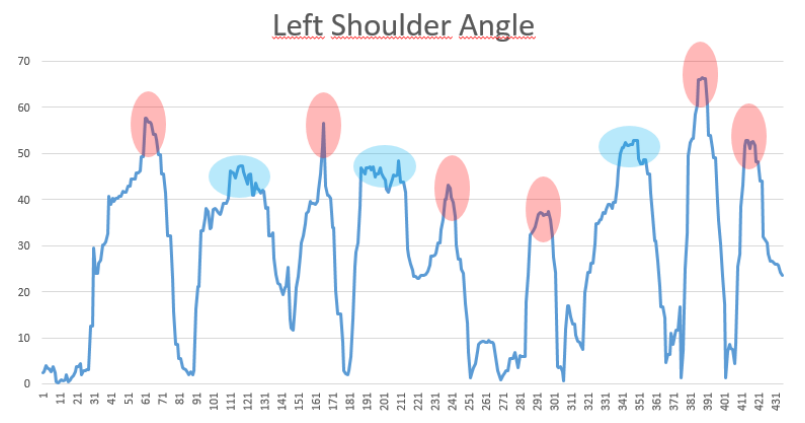

Fig. 8. ROM of the left shoulder, from the single individual, during the GesturePuzzle execution (y-axis: represents degrees of range of motion and $\mathrm{x}$-axis: represents the number of samples collected in hertz).

Increasing the feedback obtained during the execution of the task may facilitate the restoration of impaired motor ability. Moreover, the possibility of modifying the virtual set of features can make rehabilitation sessions more attractive and enjoyable. The number of physiotherapy or occupational therapy sessions, twenty sessions in four weeks of intervention [23]-[25], seems to have a positive impact on motor function improvement (in stroke patients) and allows 
the patient to be discharged from the hospital earlier, complementing their recovery at home.

Changes in the brain activity of subjects during observation, imagination, and imitation of a virtual arm that reproduces the movement of picking up and pushing a ball have also been evaluated [26]. Brain activity during observation and imagination is less intense than that recorded during imitation of the proposed movement using the virtual arm, suggesting that movement reproduction is more effective when immersed in VR environments.

\section{CONCLUSION}

We proposed here a means to evaluate the outcome of patients engaged in VR training. A pilot study with 13 healthy individuals who underwent one training session was performed, and no differences were found in brain connectivity related to the primary motor area. This result was hypothesized in advance, since we believe one session is insufficient to yield significant changes in brain synchrony, especially in healthy controls. Nevertheless, in the VRrehabilitation protocols, more training time is required, and brain connectivity alterations can show how the processes of neurorehabilitation occur. Also, measurements of the ROM of the patients were made during the training session. This information can be used both to evaluate the therapy session and as feedback to the patient, who could use it to improve his/her performance. The next step is to compare the improvement of motor function in patients who suffered a stroke when using VR therapy and the conventional approach.

\section{ACKNOWLEDGMENT}

This research was supported by FAPESP (São Paulo Research Foundation, grant 2015/23830-4) through Brazilian Institute of Neuroscience and Neurotechnology (BRAINN, FAPESP grant 2013/07559-3).

\section{DISCLOSURE STATEMENT}

No competing financial interests exist.

\section{REFERENCES}

D. M. Oliveira, S. C. Cao, X. F. Hermida, and F. M. Rodriguez, "Virtual Reality System for Industrial Training," in 2007 IEEE International Symposium on Industrial Electronics, 2007, pp. 1715-1720.

[2] B. Kenwright, "Virtual Reality: Ethical Challenges and Dangers [Opinion]," IEEE Technol. Soc. Mag., vol. 37, no. 4, pp. 20-25, Dec. 2018

[3] M. Zyda, "From visual simulation to virtual reality to games," Computer (Long. Beach. Calif)., vol. 38, no. 9, pp. 25-32, Sep. 2005.

[4] H. Sveistrup et al., "Experimental Studies of Virtual RealityDelivered Compared to Conventional Exercise Programs for Rehabilitation," CyberPsychology Behav., vol. 6, no. 3, pp. 245249, Jun. 2003.

[5] M. A. Grealy, D. A. Johnson, and S. K. Rushton, "Improving cognitive function after brain injury: the use of exercise and virtual reality.," Arch. Phys. Med. Rehabil., vol. 80, no. 6, pp. 661-7, Jun. 1999.

[6] K. E. Laver, B. Lange, S. George, J. E. Deutsch, G. Saposnik, and M. Crotty, "Virtual reality for stroke rehabilitation,"
Cochrane Database Syst. Rev., Nov. 2017.

[7] A. Turolla et al., "Virtual reality for the rehabilitation of the upper limb motor function after stroke: a prospective controlled trial.," J. Neuroeng. Rehabil., vol. 10, p. 85, Aug. 2013.

[8] A. Kubicki, F. Bonnetblanc, G. Petrement, and F. Mourey, "Motor-prediction improvements after virtual rehabilitation in geriatrics: Frail patients reveal different learning curves for movement and postural control," Neurophysiol. Clin. Neurophysiol., vol. 44, no. 1, pp. 109-118, Jan. 2014.

[9] C.-Y. Wang, W.-J. Hwang, J.-J. Fang, C.-F. Sheu, I.-F. Leong, and H.-I. Ma, "Comparison of Virtual Reality Versus Physical Reality on Movement Characteristics of Persons With Parkinson's Disease: Effects of Moving Targets," Arch. Phys. Med. Rehabil., vol. 92, no. 8, pp. 1238-1245, Aug. 2011.

[10] Z. Luo, C. K. Lim, I.-M. Chen, and S. H. Yeo, "A virtual reality system for arm and hand rehabilitation," Front. Mech. Eng. China, Dec. 2010

[11] F. D. Rose, B. M. Brooks, and A. A. Rizzo, "Virtual reality in brain damage rehabilitation: review.," Cyberpsychol. Behav., vol 8, no. 3, pp. 241-62; discussion 263-71, Jun. 2005.

[12] A. F. Brandão, D. R. C. Dias, M. P. Guimarães, L. C. Trevelin, N. A. Parizotto, and G. Castellano, "GestureCollection for Motor and Cognitive Stimuli: Virtual Reality and e-Health prospects," $J$. Heal. Informatics, vol. 10, pp. 9-16, 2018.

[13] A. F. Brandão, D. R. C. Dias, G. Castellano, N. A. Parizotto, and L. C. Trevelin, "RehabGesture: An Alternative Tool for Measuring Human Movement," Telemed. e-Health, vol. 22, no. 7, pp. 584-589, 2016.

[14] "Primesense. 3D sensors and natural interaction solutions." [Online]. Available: https://www.lib.ncsu.edu/devices/carmine109-3d-sensor.

[15] "OpenNI. Open-source sdk for 3D sensors. OpenNI." [Online]. Available: http://openni.ru/openni-sdk/.

[16] "Nite. NiTE middware libraries. Nite." [Online]. Available: http://openni.ru/files/nite/.

[17] J. Shotton et al., "Real-time human pose recognition in parts from single depth images," in CVPR 2011, 2011, pp. 1297-1304.

"Avin2. Avin2 sensor Kinect." [Online]. Available: https://github.com/avin2/SensorKinect.

[19] A. F. Brandão, N. Parizotto, and L. Trevelin, "Gesture recognition for health and rehabilitation," Motriz, vol. 21, no. 2, pp. S268-S269, 2015.

[20] B. Biswal, F. Z. Yetkin, V. M. Haughton, and J. S. Hyde, "Functional connectivity in the motor cortex of resting human brain using echo-planar MRI.," Magn. Reson. Med., vol. 34, no. 4, pp. 537-41, Oct. 1995.

[21] A. C. Evans, D. L. Collins, S. R. Mills, E. D. Brown, R. L. Kelly, and T. M. Peters, "3D statistical neuroanatomical models from 305 MRI volumes," in 1993 IEEE Conference Record Nuclear Science Symposium and Medical Imaging Conference, pp. 18131817.

[22] J. Maldjian, "WFU PickAtlas version 3.0 User Manual." [Online]. Available: http://fmri.wfubmc.edu/software/pickatlas.

[23] L. Piron et al., "Exercises for paretic upper limb after stroke: a combined virtual-reality and telemedicine approach.," J. Rehabil. Med., vol. 41, no. 12, pp. 1016-102, Nov. 2009.

[24] M. S. Cameirao, S. B. i. Badia, E. Duarte, A. Frisoli, and P. F. M. J. Verschure, "The Combined Impact of Virtual Reality Neurorehabilitation and Its Interfaces on Upper Extremity Functional Recovery in Patients With Chronic Stroke," Stroke, vol. 43, no. 10, pp. 2720-2728, Oct. 2012.

[25] A. Askin, E. Atar, H. Koçyiğit, and A. Tosun, "Effects of Kinectbased virtual reality game training on upper extremity motor recovery in chronic stroke.," Somatosens. Mot. Res., vol. 35, no. 1 , pp. 25-32, 2018.

[26] L. Holper, T. Muehlemann, F. Scholkmann, K. Eng, D. Kiper, and M. Wolf, "Testing the potential of a virtual reality neurorehabilitation system during performance of observation, imagery and imitation of motor actions recorded by wireless functional near-infrared spectroscopy (fNIRS).," J. Neuroeng. Rehabil., vol. 7, p. 57, Dec. 2010. 